\title{
Snout Beetles (Coleoptera: Curculionidae) of East Azerbaijan, Iran
}

\author{
R. RAHATI ${ }^{1}$, J. NOZARI $^{*}$ and S. KHAGHANINIA ${ }^{2}$ \\ ${ }^{1}$ Department of Plant Protection, College of Agriculture, University of Tehran, Karaj, Iran \\ ${ }^{2}$ Department of Plant Protection, Faculty of Agriculture, University of Tabriz, 51664, Tabriz, I.R. Iran
}

(Received: 4 July 2016; 12 July 2016)

\begin{abstract}
A study on the fauna of snout beetles (Coleoptera: Curculionidae) of East Azerbaijan Province (Northwest Iran) was conducted in 2014 to 2016. A total of 19 species, belonging to 4 subfamilies (Baridinae, Entiminae, Lixinae, and Molytinae) and 18 genera were collected and identified.
\end{abstract}

Keywords: Coleoptera, Curculionidae, East Azerbaijan, fauna, Iran, snout beetle.

Curculionidae (Coleoptera: Curculionoidea) is the second largest family in the animal kingdom, including about 86,000 described species worldwide. They are small to very large (1 to $60 \mathrm{~mm}$ ) in size with various habitat, shape and colour. Both adults and larvae feed on plants and the females bore into different parts of the plants where eggs are laid. This group of beetles includes a wide range of species associated with crops and stored products (Anderson, 2002; Marvaldi et al., 2005; Oberprieler et al., 2007), many of which are economically important pests. Some species are serious pests of ornamental, agricultural and forestry plants with well-known common names (for example, boll weevil, white pine weevil, strawberry root weevil and black vine weevil, etc.). Some species have become increasingly used as biological agents in the control of introduced plants (for example, Neochetina, Hylobius, Cyphocleonus and Eustenopus, etc.) (Anderson, 2002).

Although the fauna of Iranian Curculionoidea is quite speciose, it is poorly studied. The most important lists on Iranian Curculionoidea have been published by Modarres Awal (1997) and Broumand (1998); thereafter, Legalov et al. (2010) reported 61 species new for the fauna of Iran. The East Azerbaijan Province is located in the Northwest of Iran bordered clockwise by Ardabil, Zanjan and West Azerbaijan provinces. It covers an area of $45.650 \mathrm{~km}^{2}$. The climate of the province is largely influenced by the rainy winds of the Atlantic Ocean and the Mediterranean. According to existing meteorological data, local temperatures vary within the province. The highest temperature reaches 34 ${ }^{\circ} \mathrm{C}$ in July, while the lowest temperature is about $-16{ }^{\circ} \mathrm{C}$ in January. The East Azerbaijan Province encompasses vast and fertile plains, high mountains, rivers with high volume of water, vineyards, orchards, luxuriant forest and rangelands, mountain outskirts with wonderful flora, magnificent wildlife and beautiful shores around the lakes with different

* Corresponding author; e-mail: nozari@ut.ac.ir

0238-1249/\$20.00 (C) 2016 Akadémiai Kiadó, Budapest 
recreational facilities, which all together form one of the most beautiful and spectacular regions in Iran. This study provides a list of Curculionidae species in the East Azerbaijan province.

\section{Materials and Methods}

Sampling was done in different parts of East Azerbaijan province from 2014 to 2016, including Ajabshir ( $\left.37^{\circ} 28^{\prime} \mathrm{N} / 45^{\circ} 53^{\prime} \mathrm{E}\right)$, Bostan Abad ( $\left.37^{\circ} 50^{\prime} \mathrm{N} / 46^{\circ} 49^{\prime} \mathrm{E}\right)$, Hasht$\operatorname{rod}\left(37^{\circ} 28^{\prime} \mathrm{N} / 45^{\circ} 03^{\prime} \mathrm{E}\right)$, Horand ( $\left.38^{\circ} 52^{\prime} \mathrm{N} / 47^{\circ} 21^{\prime} \mathrm{E}\right)$, Jolfa $\left(38^{\circ} 52^{\prime} \mathrm{N} / 45^{\circ} 37^{\prime} \mathrm{E}\right)$, Kaleybar ( $\left.38^{\circ} 51^{\prime} \mathrm{N} / 47^{\circ} 2^{\prime} \mathrm{E}\right)$, Kandovan $\left(37^{\circ} 47^{\prime} \mathrm{N} / 46^{\circ} 14^{\prime} \mathrm{E}\right)$, Maragheh (37 $\left.23^{\prime} \mathrm{N} / 46^{\circ} 29^{\prime} \mathrm{E}\right)$, Marand ( $\left.38^{\circ} 25^{\prime} \mathrm{N} / 45^{\circ} 44^{\prime} \mathrm{E}\right)$, Mianeh ( $\left.37^{\circ} 25^{\prime} \mathrm{N} / 47^{\circ} 43^{\prime} \mathrm{E}\right)$, Sarab (37 $56^{\prime} \mathrm{N} / 47^{\circ} 32^{\prime} \mathrm{E}$ ), Shabestar $\left(38^{\circ} 10^{\prime} \mathrm{N} / 45^{\circ} 42^{\prime} \mathrm{E}\right)$, Tabriz $\left(38^{\circ} 05^{\prime} \mathrm{N} / 46^{\circ} 16^{\prime} \mathrm{E}\right)$ and Varzaqan $\left(38^{\circ} 30^{\prime} \mathrm{N} / 46^{\circ} 39^{\prime}\right.$ E). Specimens were collected using different sampling methods such as sweeping net, beating tray, aspirator, etc. (Lodos et al., 1978, 2003). All specimens were collected by the first author (Rasool Rahati) and identifications were made using the original descriptions and other main references. Classification and nomenclature of curculionids was followed as suggested by Roberto Caldara, Massimo Meregalli and Boris Korotyaev. All specimens are kept in the Jalal Afshar Zoological Museum, University of Tehran, Karaj, Iran.

\section{Results}

In this study, a total of 19 Curculionidae species from 18 genera and 4 subfamilies (Baridinae, Entiminae, Lixinae and Molytinae) were collected and identified.

\section{Subfamily Baridinae Schönherr, 1836}

Tribe Baridini Schönherr, 1836

Genus Malvaevora Zaslavskij, 1956

Malvaevora timida (Rossi, 1792)

Material examined: (1 $\sigma^{\prime}, 2$, $)$; Shabestar city, 9 May 2015

Genus Cosmobaris Casey, 1920

Cosmobaris scolopacea (Germar, 1819)

Material examined: (3 $\sigma^{\top}, 5$ q ); Hashtrod city, 23 June 2015

Genus Labiaticola Alonso-Zarazaga and Lyal, 1999

Labiaticola despicatus (Faust, 1889)

Material examined: $\left(2 \sigma^{7}\right)$; Shabestar city, 9 May 2015

Subfamily Entiminae Schönherr, 1823

Tribe Brachvderini Schönherr, 1826

Genus Epiphanops Reitter, 1895

Epiphanops persicus (Chevrolat, 1880)

Material examined: (20 ); Bostan Abad city, 14 September 2015 (20', 19);

Hashrod city, 23 June 2015 
Tribe Cyphicerini Lacordaire, 1863

Genus Ptochus Schönherr, 1826

Ptochus longicollis Boheman, 1834

Material examined: (1 $\bigcirc^{7) ; ~ A j a b s h i r ~ c i t y, ~} 8$ July 2015 (3 q); Jolfa city, 8 May 2015

Genus Chloebius Schönherr, 1826

Chloebius immeritus Schönherr, 1826

Material examined: (2ఠ', 5 \%); Jolfa city, 8 May 2015

Tribe Sciaphilini Sharp, 1891

Genus Eusomus Germar, 1824

Eusomus ovulum Germar, 1824

Material examined: (4o', 12 q); Jolfa city, 8 May 2015 (5 $\sigma^{\top}, 7$ 7 ); Marand city, 9 July 2015

Tribe Sitonini Gistel, 1848

Genus Sitona Germar, 1817

Sitona hispidulus (Fabricius, 1777)

Material examined: (3 $\left.\sigma^{7}\right)$; Kaleybar city, 5 July 2015

Sitona obsoletus (Gmelin, 1790)

Material examined: (1 $\bigcirc^{7}, 2$ ) $)$; Kaleybar city, 5 July 2015 (1 $\left.\bigcirc^{7}\right)$; Kandovan

city, 13 September 2015 (20', 4O)); Jolfa city, 8 May 2015

Tribe Tanymecini Lacordaire, 1863

Genus Chlorophanus C. R. Sahlberg, 1823

Chlorophanus flavescens (Fabricius, 1787)

Material examined: (207); Jolfa city, 8 May 2015

Tribe Polydrusini Schönherr, 1823

Genus Polydrusus Germar, 1817

Polydrusus inustus Germar, 1824

Material examined: (4ণ', 6q); Shabestar city, 9 May 2015

Genus Tanymecus Germar, 1817

Tanymecus palliatus (Fabricius, 1787)

Material examined: (1 $\left.\bigcirc^{7}\right)$; Jolfa city, 8 May 2015

Subfamily Lixinae Schönherr, 1823

Tribe Cleonini Schönherr, 1826

Genus Cleonis Dejean, 1821

Cleonis pigra (Scopoli, 1763)

Material examined: (4ণ , 4q); Bostan Abad city, 14 September 2015 (20',

3 Q ); Sarab city, 17 July 2015 (1 $q)$; Varzeqan city, 14 July 2015 (3ơ, 5 ㅇ) ;

Marand city, 9 July 2015

Genus Coniocleonus Motschulsky, 1860

Coniocleonus excoriatus (Gyllenhal, 1834)

Material examined: ( $\left.1 \bigcirc^{7}\right)$; Tabriz city, 30 April 2015

Genus Conorhynchus Motschulsky, 1860

Conorhynchus nigrivittis (Pallas, 1781) 
Material examined: (1 ð’, 1 Q); Tabriz city, 30 April 2015

Genus Cyphocleonus Motschulsky, 1860

Cyphocleonus dealbatus (Gmelin, 1790)

Material examined: (1 $\left.\sigma^{\top}, 19\right)$; Sarab city, 17 July 2015

Tribe Lixini Schönherr, 1823

Genus Larinus Dejean, 1821

Larinus turbinatus Gyllenhal, 1836

Material examined: (4ণ', 4q); Marand city, 9 July 2015

Genus Lixus Fabricius, 1801

Lixus tibialis Boheman, 1843

Material examined: (2ఠ', 1 \&); Marand city, 9 July 2015

Subfamily Molytinae Schönherr, 1823

Tribe Mecysolobini Reitter, 1913

Genus Alcidodes Marshall, 1939

Alcidodes karelinii (Boheman, 1844)

Material examined: (207); Hashrod city, 23 June 2015

\section{Discussion}

The results of this research indicated that the fauna of Curculionidae is rather diverse in the East Azerbaijan province. Among the 18 genera discussed in this paper, two species belong to the genus Sitona. Other genera have one species. Of these, 7 species are new records for East Azerbaijan Province (Epiphanops persicus, Chloebius immeritus, Ptochus longicollis, Sitona hispidulus, Tanymecus palliatus, Coniocleonus excoriatus and Conorhynchus nigrivittis).

The family Curculionidae, with over 86,000 described species, is the second largest family in the kingdom Animalia. It is self-evident that there are many other curculionid species yet to be collected in and reported from the East Azerbaijan province and of course, whole Iran; hence, it is necessary that researchers continue these faunistic works towards finding new records, and new distributional and bionomical data, especially in determining host plants and natural enemies of the Iranian Curculionidae.

\section{Acknowledgements}

The authors wish to thank Drs Boris Korotyaev, Massimo Meregalli, Roberto Caldara and Nikolai N. Yunakov for confirming the identification of collected species and Sudabe Amini (PhD Candidate University of Tehran) for his thoughtful comments.

\section{Literature}

Anderson, R. S. (2002): Curculionidae Latreille 1802. In: R. H. Arnett Jr., M. C. Thomas, P. E. Skelley and J. H. Frank (eds): American Beetles. Vol. 2, Polyphaga: Scarabaeoidea through Curculionoidea CRC Press, Boca Raton, pp. xiv+1-816. 
Broumand, H. (1998): List of Coleoptera in the Insect Collection of Plant Pests and Diseases Research Institute. J. E. S. I, Tehran, pp. 1-110.

Legalov, A. A., Ghahari, H. and Arzanov, Y. G. (2010): Annotated catalogue of Curculionid-beetles (Coleoptera: Anthribidae, Rhynchitidae, Attelabidae, Brentidae, Brachyceridae, Dryophthoridae and Curculionidae) of Iran. Amurian Zoological J. 3, 191-244.

Lodos, N., Önder, F., Pehlivan, E. and Atalay, R. (1978): Ege ve Marmara Bölgesi'nin Zararlı Böcek Faunasının Tespiti Üzerinde Çalışmalar (Curculionidae, Scarabaeidae (Coleoptera); Pentatomidae, Lygaeidae, Miridae (Heteroptera). T.C. Gıda, Tarım ve Hayvancılık Bakanlığı Zirai Mücadele ve Zirai Karantina Genel Müdürlüğü, Ankara, pp. 1-301.

Lodos, N., Önder, F., Pehlivan, E., Atalay, R., Erkin, E., Karsavuran, Y., Tezcan, S. and Aksoy, S. (2003): Faunistic studies on Curculionidae (Coleoptera) of western Black Sea central Anatolia and Mediterranean regions of Turkey. Meta Basım Matbaacılık Hizmetleri, Bornova, İzmir, pp. 1-83.

Marvaldi, A. E. and Lanteri, A. A. (2005): Key to higher taxa of South American weevils based on adult characters (Coleoptera, Curculionoidea). Revista Chilena de Historia Natural 78, 65-87.

Modarres Awal, M. (1997): Family Curculionidae. In: M. Modarres Awal (ed.): List of Agricultural Pests and their Natural Enemies in Iran. Ferdowsi University Press, Mashhad, pp. 319-335.

Oberprieler, R. G., Marvaldi, A. E. and Anderson, R. S. (2007): Weevils, weevils, weevils everywhere. Zootaxa $1668,491-520$. 